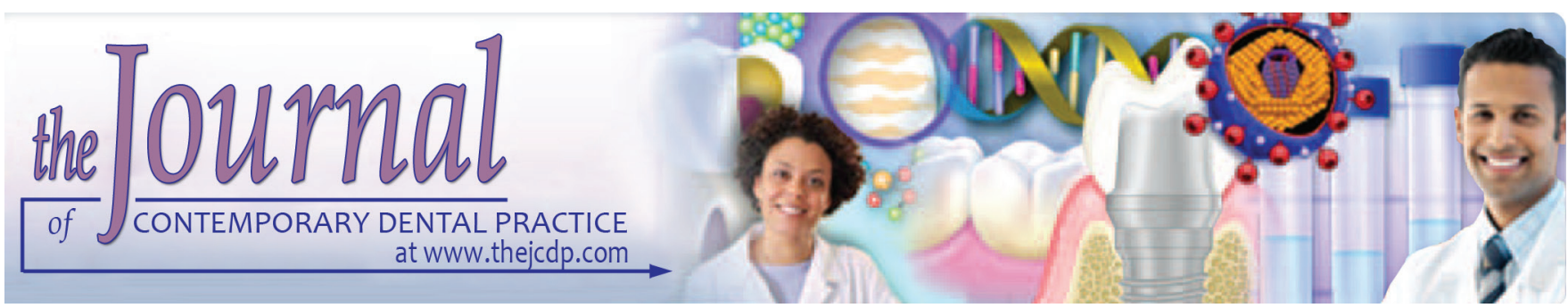

\title{
Impact of Mouthwashes on Antibacterial Activity of Subjects with Fixed Orthodontic Appliances: A Randomized Clinical Trial
}

${ }^{1}$ A Nishad, ${ }^{2}$ NS Sreesan, ${ }^{3}$ Joseph Joy, ${ }^{4}$ Lakshmi Lakshmanan, ${ }^{5}$ Joyce Thomas, ${ }^{6}$ VA Anjali

\begin{abstract}
Aim: The study aimed to assess the impact of mouthwashes on antibacterial activity of individuals with fixed orthodontic appliances.

Materials and methods: A total of 60 individuals were considered in the study. Sixty (20 each group) nonextraction class I individuals were randomly divided into experimental and control groups. Group I: Experimental group [chlorhexidine (CHX) mouthwash], group II: Experimental group (neem mouthwash), group III: Control group (distilled water). All the clinical examinations were done at baseline and 30th day respectively, after the start of orthodontic treatment. The mean differences between the different experimental groups were calculated using one-way analysis of variance (ANOVA) test.
\end{abstract}

Results: There was no statistical significance at baseline mean plaque index (PI), gingival index (GI) scores, and Streptococcus mutans (SM) colony count between groups. The $\mathrm{PI}$ and $\mathrm{GI}$ scores among $\mathrm{CHX}$ and neem mouthwash groups $(p=0.002$, $p=0.032$ respectively) were significantly reduced after intervention and also the SM colonies count was reduced in $\mathrm{CHX}$ and neem mouthwash groups and there was significant difference between the groups.

\footnotetext{
${ }^{1}$ Department of Orthodontics and Dentofacial Orthopedics Noorul Islam College of Dental Science, Thiruvananthapuram Kerala, India

${ }^{2}$ Department of Orthodontics and Dentofacial Orthopedics KMCT Dental College, Kozhikode, Kerala, India

${ }^{3}$ Department of Conservative Dentistry and Endodontics, Mar Baselios Dental College, Kothamangalam, Kerala, India

${ }^{4,6}$ Department of Orthodontics and Dentofacial Orthopedics Malabar Dental College \& Research Centre, Edappal, Kerala, India

${ }^{5}$ Department of Prosthodontics, Al-Azhar Dental College Thodupuzha, Kerala, India

Corresponding Author: A Nishad, Department of Orthodontics and Dentofacial Orthopedics, Noorul Islam College of Dental Science, Thiruvananthapuram, Kerala, India, e-mail: drnishath@ hotmail.com
}

Conclusion: As both mouthwashes showed significant effectiveness on antibacterial activity in individuals with fixed orthodontic appliances, neem mouthwash can be used as an alternative to $\mathrm{CHX}$.

Clinical significance: It is better to have a sound knowledge regarding the use of mouthwash in long term as fixed orthodontics are associated with accumulation of SM, enamel demineralization, and an increased number of carious lesions, predominantly in sites adjacent to bracket.

Keywords: Chlorhexidine, Neem, Orthodontic appliances, Streptococcus mutans.

How to cite this article: Nishad A, Sreesan NS, Joy J, Lakshmanan L, Thomas J, Anjali VA. Impact of Mouthwashes on Antibacterial Activity of Subjects with Fixed Orthodontic Appliances: A Randomized Clinical Trial. J Contemp Dent Pract 2017;18(12):1112-1116.

\section{Source of support: Nil}

Conflict of interest: None

\section{INTRODUCTION}

Practicing satisfactory dental hygiene is one of the main challenges that orthodontic patients face. Failure to maintain this makes these patients vulnerable to dental caries. There is change in the oral cavity homeostasis, such as changes in the salivary $\mathrm{pH}$ and the composition of the oral flora and consequently increase in the dental plaque accumulation; this is because fixed orthodontic appliances inhibit effective tooth brushing and cause potential food retention. ${ }^{1}$

There is unpredictable effect of orthodontic treatment on the nonvisible part of the oral cavity, i.e., the microbiome. The clinical parameters easily indicate the possible changes in the oral microbiome during orthodontic treatment. The placement of fixed orthodontic appliances more likely led to impaired gingival health status and 
increased plaque formation. ${ }^{2}$ Formation of white spot lesions, undesirable esthetic effect, and cavity formation requiring restoration can also occur as a result. ${ }^{3}$

The initial step in dental caries is dental plaque formation, and SM is considered as the main culprit in this multispecies dental biofilm. There is fivefold increase in the number of SM during orthodontic treatment as has been observed. ${ }^{4}$

Mouthwashes reduce microbial plaques. To reduce dental plaques and pathogenic microorganisms, such as SM among the available mouthwashes, $\mathrm{CHX}$ was shown to be highly effective. In most of the recent studies on mouthwashes, gold standard is taken as CHX, and to compare the efficacy of other products, it is used as positive control. However, the side effects, such as discoloration of tooth, distaste, uncomfortable sensation, such as dryness, and burning sense in the mouth avoid patients from using this mouthwash. ${ }^{5}$

Neem as a mouthwash effectively inhibits both Grampositive and Gram-negative organisms that include Salmonella, Escherichia coli, and Streptococcus. Extracts of neem inhibit the growth of SM by their antioxidant, anti-inflammatory, and antimicrobial properties; hence, they are used for the treatment of periodontitis. ${ }^{6}$ Hence, the present study was conducted to assess the impact of mouthwashes on antibacterial activity of individuals with fixed orthodontic appliances.

\section{MATERIALS AND METHODS}

Eighteen to 35-year-old individuals with newly started fixed orthodontic treatment (Fig. 1) in the Department of Orthodontics, Noorul Islam College of Dental Sciences, Thiruvananthapuram, Kerala, India, were taken into the study. Total of 60 individuals were considered for the study. Institutional Review Board issued ethical clearance and patients were explained about the procedure

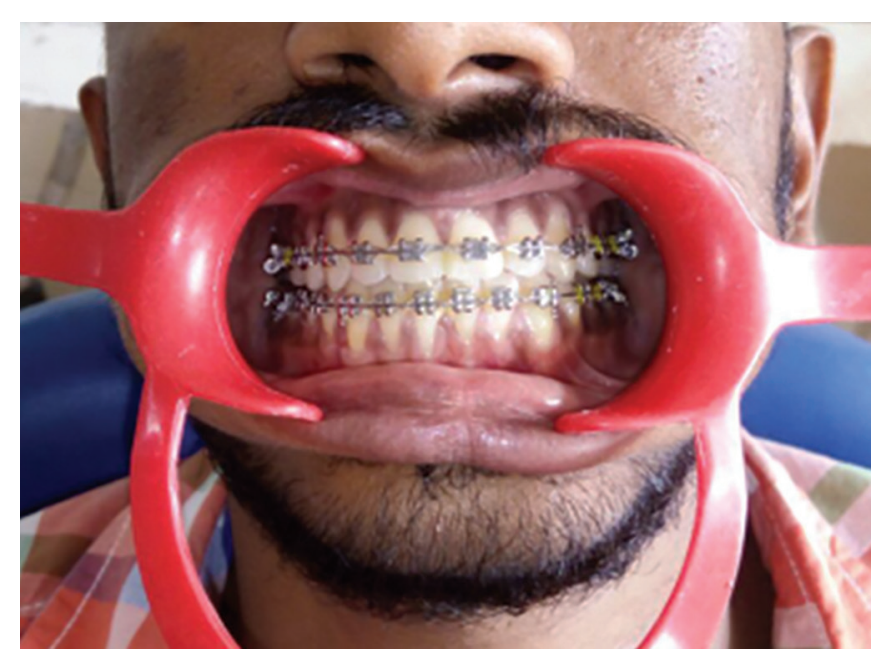

Fig. 1: Individual with fixed orthodontic appliance and consent was taken from all participating individuals. Inclusion criteria were individuals with complete dentition up to the second molar, no associated comorbidities, no recent use of antibiotic or antibacterial mouthwash in recent past (1 month), no caries, or demineralization. Exclusion criteria were individuals on oral antimicrobials or antibiotic within the past 3 months and individuals with prosthodontic appliances.

Individuals were randomly divided into three experimental and control groups from 60 (20 in each group) nonextraction class I individuals.

- Group I: Experimental group (CHX mouthwash) included 20 individuals with $\mathrm{CHX}$ mouthwash and they were instructed to use $5 \mathrm{~mL}$, two times a day after meals, and all the clinical examinations were done at baseline and 30th day respectively, after the start of orthodontic treatment.

- Group II: Experimental group (neem mouthwash) included 20 individuals with neem mouthwash and they were instructed to use $5 \mathrm{~mL}$, two times a day after meals, and all the clinical examinations were done at baseline and 30th day respectively, after the start of orthodontic treatment.

- Group III: Control group (distilled water) included 20 individuals with distilled water and they were instructed to use $5 \mathrm{~mL}$, two times a day after meals, and all the clinical examinations were done at baseline and 30th day respectively, after the start of orthodontic treatment.

After bonding, all individuals were provided with Oral B orthodontic toothbrushes and toothpaste. All the individuals were instructed to follow horizontal-scrub method to brush their teeth at least twice a day for 2 minutes. During 1 hour, after using the mouthwashes, the participants were told to refrain from any food or drink.

\section{Clinical Examination}

\section{Plaque Index}

The site is said to be positive if plaque deposits were found when a periodontal probe was circulated between the bracket base and free gingival margin at six sites around each tooth.

\section{Gingival Index}

A William's periodontal probe was inserted into the gingival sulcus at an angle of $45^{\circ}$ and minimal pressure running the probe from interproximal to interproximal along the buccal and lingual aspects of all teeth (Fig. 2). The site was said to be positive after probing if it bleeds within 10 seconds. 


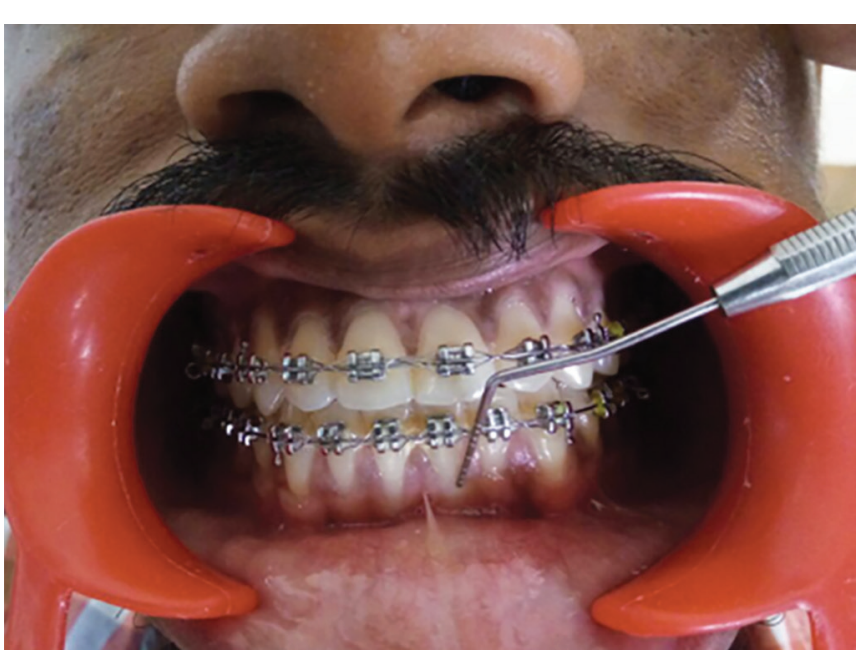

Fig. 2: Gingival index examination for individual with fixed orthodontic appliance

\section{Streptococcus mutans Count ${ }^{7}$}

About $3 \mathrm{~mL}$ of serially diluted transport fluid (VMG II) was used where saliva $(1 \mathrm{~mL})$ was placed, and all samples smeared to mitis salivarius bacitracin agar (mitis salivarius agar), Chapman Tellurite solution, 150 gm sucrose, and $200 \mathrm{U} / \mathrm{mL}$ bacitracin. The colonies were counted after incubation at $37^{\circ} \mathrm{C}$ for 48 hours in candle jars. It was divided into:

- Low (<105 colony-forming unit (CFU)/mL)

- Moderate (105-106 CFU/mL)

- High ( $\geq 106 \mathrm{CFU} / \mathrm{mL})$.

\section{Statistical Analysis}

Software used for the analysis was Statistical Package for the Social Sciences, version 16. One-way ANOVA test was used to calculate the mean difference between the different experimental groups. The level of significance was set at $5 \%$.

\section{RESULTS}

Table 1 reveals no significant difference between the groups $(p=0.92)$ as mean PI scores between all three groups at baseline show almost similar to mean plaque scores.

Table 2 shows that the mean GI score of neem mouthwash was slightly higher $(0.95 \pm 0.25)$ than CHX and distilled water groups. However, it was not statistically significant between the groups $(\mathrm{p}=0.373)$.

There was no significant difference between the groups (Table 3) in the SM colonies count; it was less in all the three groups.

Table 4 shows that the mean PI score was increased in the distilled water group $(1.09 \pm 0.57)$ than $\mathrm{CHX}$ and neem mouthwash groups after intervention. In addition, there was a significant difference found between the groups $(\mathrm{p}=0.002)$.

Table 5 reveals that the mean GI score of CHX mouthwash significantly $(p=0.032)$ reduced than neem and distilled water groups.

Table 6 shows that the SM colonies count was reduced in CHX and neem mouthwash groups and there was significant difference between the groups.

\section{DISCUSSION}

Fixed orthodontic appliances make the patients vulnerable to plaque accumulation around brackets and gingival margins. Application of mouthwashes has been suggested as the supplement of oral hygiene practices as mechanical

Table 1: Comparison of mean PI scores between groups at baseline

\begin{tabular}{lllllll}
\hline Groups & $n$ & Mean Pl score & Standard deviation & Standard error & $f$-value & $p$-value \\
\hline Chlorhexidine & 20 & 1.2000 & 0.57400 & 0.12835 & 0.084 & 0.92 \\
Neem & 20 & 1.2400 & 0.46158 & 0.10321 & & \\
Distilled water & 20 & 1.1700 & 0.58319 & 0.13040 & \\
\hline Total & 60 & 1.2033 & 0.53392 & 0.06893 & \\
\hline
\end{tabular}

Table 2: Comparison of mean GI score between groups at baseline

\begin{tabular}{lllllll}
\hline Groups & $n$ & Mean Gl score & Standard deviation & Standard error & $f$-value & $p$-value \\
\hline Chlorhexidine & 20 & 0.8050 & 0.36631 & 0.8191 & 1.003 & 0.373 \\
Neem & 20 & 0.9500 & 0.25854 & 0.05781 & \\
Distilled water & 20 & 0.8368 & 0.38328 & 0.08793 & \\
\hline Total & 60 & 0.8644 & 0.33976 & 0.04423 & \\
\hline
\end{tabular}

Table 3: Comparison of mean SM count between the groups at baseline

\begin{tabular}{lllllll}
\hline Groups & $n$ & Mean SM score & Standard deviation & Standard error & $f$-value & $p$-value \\
\hline Chlorhexidine & 20 & 103.55 & 1.761 & 0.394 & 0.140 & 0.870 \\
Neem & 20 & 103.85 & 1.814 & 0.406 & \\
Distilled water & 20 & 103.65 & 1.899 & 0.425 & \\
\hline Total & 60 & 103.68 & 1.799 & 0.232 & \\
\hline
\end{tabular}


Impact of Mouthwashes on Antibacterial Activity of Subjects with Fixed Orthodontic Appliances

Table 4: Comparison of mean PI scores between groups after intervention

\begin{tabular}{|c|c|c|c|c|c|}
\hline Groups & $n$ & Mean $\pm S D$ & $\begin{array}{l}\text { Standard } \\
\text { error }\end{array}$ & f-value & $p$-value \\
\hline Chlorhexidine & 20 & $0.5350 \pm 0.32650$ & 0.07301 & 7.234 & 0.002 \\
\hline Neem & 20 & $0.8800 \pm 0.45952$ & 0.10275 & & \\
\hline Distilled water & 20 & $1.0900 \pm 0.57756$ & 0.12915 & & \\
\hline Total & 60 & $0.8350 \pm 0.51283$ & 0.06621 & & \\
\hline
\end{tabular}

SD: Standard deviation
Table 5: Comparison of mean GI scores between groups after intervention

\begin{tabular}{|c|c|c|c|c|c|}
\hline Groups & $n$ & Mean $\pm S D$ & $\begin{array}{l}\text { Standard } \\
\text { error }\end{array}$ & $f$-value & $p$-value \\
\hline Chlorhexidine & 20 & $0.3950 \pm 0.34713$ & 0.07762 & 3.67 & 0.032 \\
\hline Neem & 20 & $0.6200 \pm 0.34580$ & 0.07732 & & \\
\hline Distilled water & 20 & $0.6850 \pm 0.37173$ & 0.08312 & & \\
\hline Total & 60 & $0.5667 \pm 0.37083$ & 0.04787 & & \\
\hline
\end{tabular}

Table 6: Comparison of mean SM count between the groups after intervention

\begin{tabular}{lllllll}
\hline Groups & $n$ & Mean SM score & Standard deviation & Standard error & $f$-value & $p$-value \\
\hline Chlorhexidine & 20 & 93.95 & 6.320 & 1.413 & 13.540 & 0.0001 \\
Neem & 20 & 96.30 & 5.449 & 1.218 & \\
Distilled water & 20 & 102.05 & 2.704 & 0.605 & \\
\hline Total & 60 & 97.43 & 6.046 & 0.781 & \\
\hline
\end{tabular}

tools seem to be not enough in controlling dental plaque in these patients. ${ }^{8}$

Many antiplaque agents are available in the market. Due to the rise in bacterial resistance to antibiotics, there is raising interest in the development of other classes of antimicrobials to treat the infection. Increased efforts are made to explore products from Ayurveda, the traditional system of medicine in India. The history of knowledge about dantamulagatarogas in Ayurveda is traced back to the period of Sushruta, and regarding these diseases we may consider eight dantamulagataroga. Many natural herbs were used against various oral health problems, such as bleeding gums, halitosis, mouth ulcers, and decay either using alone or in combination which is scientifically proven to be safe and effective. ${ }^{9}$

In this study, the PI and GI scores were significantly reduced by neem mouthwash. The neem mouthwash was used in this study as the taste should not be a hindrance for its use with maximal inhibition of bacteria and plaque. According to Botelho et al, ${ }_{1}^{10}$ an indica-based mouth rinse can be used as an alternative treatment for periodontal diseases because of its high efficacy and is in accordance with our present study. There was reduction in the probing pocket depth and gain in the clinical attachment level by the use of neem extract as noted by Patel and Venkatakrishna-Bhatt. ${ }^{11}$

Although neem and CHX mouthwashes reduced the number of SM colonies significantly, neem seems to be less potent than CHX. There are only few known studies that delineate the difference between the effect of $\mathrm{CHX}$ and neem on the SM colonies in microbial plaque quantitatively. Mortazavi and Balali ${ }^{12}$ reported that both $\mathrm{CHX}$ and Persica mouthwashes reduced the number of SM colonies to an insignificantly different level.

There was no significant difference in a study done by Saffari et $\mathrm{al}^{4}$ between the two groups before using mouthwashes, which implies that both groups are similar with respect to the status of SM colonization. Between the first and second steps within CHX group $(\mathrm{p}=0.001)$. There was a significant difference found in SM colonization. Chlorhexidine mouthwash is very effective in the reduction of microbial plaque. Suppression of Streptococci counts depends on the coating of $\mathrm{CHX}$ to the tooth surfaces. Because of orthodontic brackets, it might prevents the effective function of CHX in all surfaces on SM.

Several studies ${ }^{13,14}$ have shown that after treatment with highly concentrated CHX, SM are effectively inhibited for a considerably long time. Chlorhexidine was shown to be effective in decreasing Streptococci colonization and also in decreasing dental caries as shown in a study by Maltz et $\mathrm{al}^{15}$ on individuals who were not orthodontic patients.

Khan and Virginia ${ }^{16}$ stated that implementing proper oral hygiene in patients with fixed orthodontic appliances is difficult as it contributes to plaque accumulation and the results were consistent with the results of this study. The $\mathrm{pH}$ level is lowered and the demineralization of tooth hard tissue around their bases is due to brackets. The number of SM increases in the oral cavity after orthodontic bonding. Food traps because of the placement of orthodontic appliances can cause gingivitis and periodontal destruction.

In a study conducted by Salehi and Danaie, ${ }^{17}$ there was no significant difference in the number of SM colonies from the groups before treatment. This indicates that the mouthwashes were responsible for change in the number of bacterial colonies. Positive control in this study was CHX. The numbers of SM colonies were significantly reduced by both mouthwashes at T2 and T3, but Persica (neem) was less potent than CHX. These results show similarity to the present study and also with other studies Renton-Harper et $\mathrm{al}^{18}$ and Almas. ${ }^{19}$ 
Neem mouthwash can be an alternative in low socioeconomic status population as it is cost-effective as compared with the cost of $0.2 \% \mathrm{CHX}$ gluconate mouthwash. In addition, $0.2 \% \mathrm{CHX}$ gluconate mouthwash causes brown staining of teeth in individuals using them, which was not observed in neem mouthwash individuals. It was also evident that tooth discoloration or unpleasant taste was more common in CHX group than neem mouthwash group. Therefore, neem mouthwash is more preferable to $0.2 \%$ CHX gluconate mouthwash. ${ }^{20}$

A study done by Krishnan et $\mathrm{al}^{21}$ reported that there is an alteration in the oral hygiene habits and periodontal health and also the qualitative nature of the local bacterial biofilm is changed after the placement of an orthodontic appliance in a patient's mouth.

To eliminate the bias of variable host response, a crossover design with washout period would have been more authenticating. Therefore, it is advisable to test the antiplaque and antigingivitis efficacy of these mouthwashes in a long-term period in future studies, which can also help us to know more about the advantages and disadvantages of these mouthwashes.

\section{CONCLUSION}

Neem mouthwash can be used as an alternative to CHX as both mouthwashes show a significant reduction in the SM level. It also suggests that incidence of tooth discoloration or unpleasant taste is less common with neem than with $\mathrm{CHX}$. A larger long-term clinical trial is needed to explore the antibacterial efficacy of the mouthwashes.

\section{REFERENCES}

1. Peros K, Mestrovic S, Anic-Milosevic S, Rosin-Grget K, Slaj M. Antimicrobial effect of different brushing frequencies with fluoride toothpaste on Streptococcus mutans and Lactobacillus species in children with fixed orthodontic appliances. Korean J Orthod 2012 Oct;42(5):263-269.

2. Gong Y, Lu J, Ding X. Clinical, microbiologic, and immunologic factors of orthodontic treatment-induced gingival enlargement. Am J Orthod Dentofacial Orthop 2011 Jul;140(1):58-64.

3. Koopman JE, van der Kaaij NC, Buijs MJ, Elyassi Y, van der Veen MH, Crielaard W, ten Cate JM, Zaura E. The effect of fixed orthodontic appliances and fluoride mouthwash on the oral microbiome of adolescents-a randomized controlled clinical trial. PLoS One 2015;10(9):e0137318.

4. Saffari F, Danesh Ardakani M, Zandi H, Heidarzadeh H, Moshafi MH. The effects of chlorhexidine and Persica mouthwashes on colonization of Streptococcus mutans on fixed orthodontics o-rings. J Dent (Shiraz) 2015 Mar;16(1):54-57.

5. Rosin M, Welk A, Bernhardt O, Ruhnau M, Pitten FA, Kocher T, Kramer A. Effect of a polyhexamethylene biguanide mouthrinse on bacterial counts and plaque. J Clin Periodontol 2001 Dec;28(12):1121-1126.
6. Jalaluddin M, Rajasekaran UB, Paul S, Dhanya RS, Sudeep CB, Adarsh VJ. Comparative evaluation of neem mouthwash on plaque and gingivitis: a double-blind crossover study. J Contemp Dent Pract 2017 Jul;18(7):567-571.

7. Lombardo L, Ortan YÖ, Gorgun Ö, Panza C, Scuzzo G, Siciliani G. Changes in the oral environment after placement of lingual and labial orthodontic appliances. Prog Orthod 2013;14(1):28.

8. Singh A, Daing A, Dixit J. The effect of herbal, essential oil and chlorhexidine mouthrinse on de novo plaque formation. Int J Dent Hyg 2013 Feb;11(1):48-52.

9. Narayan A, Mendon C. Comparing the effect of different mouthrinses on de novo plaque formation. J Contemp Dent Pract 2012 Jul;13(4):460-463.

10. Botelho MA, dos Santos RA, Martins JG, Carvalho CO, Paz MC, Azenha C, Ruela RS, Queiroz DB, Ruela WS, Marinho G, et al. Efficacy of a mouthrinse based on leaves of the neem tree (Azadirachta indica) in the treatment of patients with chronic gingivitis: A double-blind, randomized, controlled trial. J Med Plants Res 2008 Nov;2(11):341-346.

11. Patel VK, Venkatakrishna-Bhatt H. Folklore therapeutic indigenous plants in periodontal disorders in India (review, experimental and clinical approach). Int J Clin Pharmacol Ther Toxicol 1988 Apr;26(4):176-184.

12. Mortazavi M, Balali N. Intra oral effect of Persica mouthwash, irsha, povidone iodine $1 \%$, chlorhexidine and hypertonic chloride sodium on the rate of mutans streptococci of saliva, School of Dentistry, Shiraz University of Medical Sciences, Dentistry Doctoral Dissertation; 2001. p. 797.

13. Attin R, Tuna A, Attin T, Brunner E, Noack MJ. Efficacy of differently concentrated chlorhexidine varnishes in decreasing mutans Streptococci and Lactobacilli counts. Arch Oral Biol 2003;48(7):503-509.

14. Ie YL, Schaeken MJ. Effect of single and repeated application of chlorhexidine varnish on mutans streptococci in plaque from fissures of premolar and molar teeth. Caries Res 1993;27(4):303-306.

15. Maltz M, Zickert I, Krasse B. Effect of intensive treatment with chlorhexidine on number of Streptococcus mutans in saliva. Scand J Dent Res 1981;89(6):445-449.

16. Khan R, Virginia V. Efficacy of chlorhexidine varnish on Streptococcus mutans in plaque. Orthod Cyber J 2010 Mar:1-10.

17. Salehi P, Danaie M. Comparison of the antibacterial effects of Persica mouthwash with chlorhexidine on Streptococcus mutans in orthodontic patients. DARU J Pharm Sci 2006;14(4): 178-182.

18. Renton-Harper P, Addy M, Mora NJ, Doherty FM, Newcombe RG. A comparison of chlorhexidine, cetylpyridinium chloride, triclosan and C31G mouthrinse products for plaque inhabitation. J Periodontol 1996 May;67(5):486-489.

19. Almas K. The effect of Salvadora persica extract (miswak) and chlorhexidine gluconate on human dentin: A SEM study. J Contemp Dent Pract 2002 Aug;3(3):27-35.

20. Parwani SR, Parwani RN, Chitnis PJ, Dadlani HP, Prasad SV. Comparative evaluation of anti-plaque efficacy of herbal and $0.2 \%$ chlorhexidine gluconate mouthwash in a 4 -day plaque re-growth study. J Indian Soc Periodontol 2013 Jan;17(1): 72-77.

21. Krishnan V, Ambili R, Davidovitch Z, Murphy NC. Gingiva and orthodontic treatment. Semin Orthod 2007;13:257-271. 